\title{
Other Edition
}

National Cancer Institute

\section{Source}

National Cancer Institute. Other Edition. NCI Thesaurus. Code C159627.

An indication that the edition is other than those listed. 